\title{
Gaze allocation in a dynamic situation: Effects of social status and speaking
}

\author{
Tom Foulsham*, Joey T. Cheng, Jessica L. Tracy, Joseph Henrich, Alan Kingstone \\ Department of Psychology, University of British Columbia, Canada
}

\section{A R T I C L E I N F O}

\section{Article history:}

Received 11 February 2010

Revised 9 September 2010

Accepted 24 September 2010

\section{Keywords:}

Eye movements

Social attention

Social status

Speech perception

Dynamic scene perception

\begin{abstract}
A B S T R A C T
Human visual attention operates in a context that is complex, social and dynamic. To explore this, we recorded people taking part in a group decision-making task and then showed video clips of these situations to new participants while tracking their eye movements. Observers spent the majority of time looking at the people in the videos, and in particular at their eyes and faces. The social status of the people in the clips had been rated by their peers in the group task, and this status hierarchy strongly predicted where eyetracker participants looked: high-status individuals were gazed at much more often, and for longer, than low-status individuals, even over short, 20-s videos. Fixation was temporally coupled to the person who was talking at any one time, but this did not account for the effect of social status on attention. These results are consistent with a gaze system that is attuned to the presence of other individuals, to their social status within a group, and to the information most useful for social interaction.
\end{abstract}

(c) 2010 Elsevier B.V. All rights reserved.

\section{Introduction}

Human environments have three defining characteristics that are often neglected by researchers investigating visual attention. First, they are very complex, requiring a gaze orienting system evolved to concentrate resources on the most informative objects at the expense of others. This system emerges as a natural consequence of the complexity of the environment and the existence of a foveated visual system: rather than perceiving everything in the visual field with equal fidelity, humans possess a central region of high-acuity which they shift to select items for more extensive processing. Thus, although attention research has traditionally been concerned with covert orienting to stimuli in simple arrays, investigations of attention in natural behaviour have relied increasingly on the measurement of eye movements (Findlay \& Gilchrist, 2003; Hayhoe \& Ballard, 2005). In particular, this field of inquiry seeks to identify the stimuli that are likely to attract eye fixations in different conditions. In some circumstances,

\footnotetext{
* Corresponding author. Address: Department of Psychology, 2136 West Mall, Vancouver, B.C., Canada V6T 1 Z4.

E-mail address: tfoulsham@psych.ubc.ca (T. Foulsham).
}

these stimuli may be best described by their low level features-salient items such as a bright object on a dark background are particularly likely to be fixated (Foulsham \& Underwood, 2007; Itti \& Koch, 2000). However, in more realistic and complex situations, where people look is closely related to their actions, goals and cognitions in each environmental context (Ballard \& Sprague, 2005; Land \& Hayhoe, 2001; Yarbus, 1967).

A second defining characteristic is that, for humans, this environmental context tends to be social. More often than not, humans are immersed in an environment that includes other people, and a useful, and perhaps fundamental, goal of attention is to keep track of these individuals. Social attention allows people to monitor the behaviour, intentions and emotions of others, in order to guide their own actions, interactions, and learning processes. In laboratory studies, this phenomenon has been studied by showing that the faces, and in particular the eyes, of other people are salient items and powerful attentional cues. For example, schematic eyes direct attention reflexively in a manner thought to correspond to "gaze following" (Friesen \& Kingstone, 1998). In images of complex natural scenes, viewers spend a large and disproportionate amount of time fixating other people, and in particular the eyes of others 
(Birmingham, Bischof, \& Kingstone, 2008). Children and adults with autistic spectrum disorder, who show abnormal and reduced social interactions, may not look at people in scenes and movies to the same degree as normally functioning participants (Dalton et al., 2005; Klin, Jones, Schultz, Volkmar, \& Cohen, 2002), and these deficits in social attention may even be a causative factor in the disorder (Baron-Cohen, 1995).

Third, the natural environment is highly dynamic because the state, location and salience of the objects within it change over time. Many laboratory studies of visual attention are concerned with how people select items in space (for example targets in a search task) and the goals, stimuli and locations in these studies typically remain fixed (although some paradigms do require more dynamic attentional selection, e.g. multiple object tracking, Pylyshyn \& Storm, 1988; the attentional blink, Raymond, Shapiro, \& Arnell, 1992; task switching, Rogers \& Monsell, 1995). The guidance of eye movements in natural scenes is often studied using static images (Foulsham \& Underwood, 2008; Henderson, 2003), but it is not always clear how well this research transfers to the real world, where individuals and the visual environment are often moving, and where particular objects need to be fixated at certain times. In contrast, studies of gaze allocation in real world activities have typically emphasized the temporal patterning of eye movements in relation to action (Land \& Hayhoe, 2001). For example, people look toward an object a few seconds before manipulating it, and they then move their gaze to the next task in the sequence. Recently, some research has explored the distribution of attention and eye movements in movies, and these experiments have suggested that people show a relatively high degree of convergence in cognitive processing and the distribution of attention (Hasson, Nir, Levy, Fuhrmann, \& Malach, 2004). In movies, gaze seems to be drawn to both low-level salient cues (such as suddent onsets and movement: Itti, 2005) and to semantic (whilst not necessarily salient) stimuli such as meaningful events and the actions of others (Klin et al., 2002).

In this paper we investigate gaze allocation in a set of video clips showing three individuals conversing. Where and when do people look when naturally viewing such clips? While these are relatively controlled stimuli, they contain real people embedded in a realistic background and a dynamic situation, allowing an exploration of the spatiotemporal distribution of attention in a social context. Previous research would predict that the people in the clips will be potent at drawing the attention of observers, even though there is no particular task requirement to fixate them. Which factors will determine who gets fixated, and when? The use of complex stimuli with several people adds a social dimension and permits us to investigate whether social psychological constructs have an effect on the allocation of eye movements.

One social factor that may be critical is the social status of the different individuals in the environment. In almost all social situations, humans readily develop hierarchically structured relationships, with some individuals exerting more influence on others and, consequently, attaining in- creased access to reproductively relevant resources (e.g., food, mates; Berger, Rosenholtz, \& Zelditch, 1980). Indeed, individual differences in social status or rank may be ubiquitous in human social interactions (Boehm, 1993). Many other primates also form strong social hierarchies, and gaze following has been documented in several of these, such as monkeys (Emery, 2000). Ring-tailed lemurs also show spontaneous gaze following of other social group members in their natural environment, suggesting that social attention evolved early in species that interact in social groups (Shepherd \& Platt, 2008). Chance (1967) hypothesized that social attention would reflect the dominance hierarchy of primate groups, such that the dominant individual receives the greatest number of glances, and a recent study of patas monkeys supported this prediction (McNelis \& Boatright-Horowitz, 1998). It has also been demonstrated that the effectiveness of gaze as a social cue depends on the relative social status of the individual: low status monkeys reflexively follow the gaze of any familiar monkey, but high-status macaques will only respond in this way to other high-status animals (Shepherd, Deaner, \& Platt, 2006).

In humans, observational studies have documented rank-biased attention among children, by coding their apparent gaze (Abramovitch, 1976; LaFreniere \& Charlesworth, 1983; Vaughn \& Waters, 1981). However, experimental evidence for effects of social status on attention in humans is scarce; similarly, very few studies have used eye-tracking methodology to assess the impact of status on humans' attention. One recent study reported that the social status of people depicted in an array of photographs influenced the extent to which these individuals attracted attention (Maner, DeWall, \& Gailliot, 2008): the frequency of high-status males in an array was over-estimated, and an eye tracking study confirmed that people spent more time looking at men who were rated as high status. This is consistent with evolutionary theories positing that social status is important in mate selection, particularly for women choosing a male partner. However, consistent with evolutionary approaches predicting the importance of attention to high-status individuals for reasons other than mate choice (Henrich \& Gil-White, 2001), high-status males were also potent in attracting the attention of male observers.

Although these findings suggest that the social status of targets in a display may influence the amount of attention they receive, they are also somewhat limited. Maner et al. manipulated social status by editing photographs to show individuals wearing either professional or casual attire, and their stimuli were static photographs isolated on a blank screen with no social context, no movement, and a task that placed few demands on the attentional system. In contrast, here we measure gaze while observers watch video clips of a real social interaction, and social status is quantified on the basis of previous ratings made by peers who participated in the interaction. If social status affects the distribution of gaze in this study, it will provide evidence: (i) that attention is guided, top-down, by social attributions rather than just by feature salience and (ii) that social status plays a role in early human information processing. 


\section{Methods}

\subsection{Participants}

Twenty-five students participated in the experiment. All were recruited through the University of British Columbia Human Subject Pool, and they gave their full informed consent and received course credit in return for participating. All participants had normal vision and did not wear glasses. After the experiment, it was confirmed that the participants were unfamiliar with the people they viewed in the experimental video clips.

\subsection{Stimuli and design}

The experimental stimuli consisted of four sets of video clips. Each set was derived from a previous experiment (Cheng, Tracy, Henrich, Foulsham, \& Kingstone, in preparation) in which groups of unacquainted undergraduates completed an interactive decision-making task while being recorded by an unconcealed high-definition video camera with built-in microphone positioned in front of them. The decision-making task concerned a hypothetical situation requiring participants to rank a list of items for their use in a survival situation (i.e. "which items would your group need to survive if marooned on the moon?"). Participants were given 20 min to discuss this task in groups of 6 , sitting around a table with three people on each side, before deciding on a group answer. To incentivize correct responses, participants knew that if the group's final response was close to the correct answer, each participant would be given a monetary bonus. The videos used in the present research featured the three individuals on one side of the table. Fig. 1 depicts a schematic of the scene and the layout of the resulting video frames.

Four representative videos were chosen for the eye tracking study. In each case, the three individuals in the video were classified according to social status scores from the original group-interaction experiment (from now on we will refer to these three individuals as the "targets"). Specifically, in that previous study, after the group task all group members rated the social status and influence of each target, among a battery of other judgments (three items on a 7-point scale, e.g., "this person led the task"). Ratings were made in a round-robin fashion then aggregated across peers. The four sets of clips used were chosen because peer-rated scores revealed clear relative status differences of the targets within them; on average there was a 2.5 point difference in mean status ratings (overall $\mathrm{SD}=1.4$ ) between two of the targets, with the third falling in between, suggesting that these individuals could be considered high, low and medium status. Given these differences, in subsequent analyses we were able to compare the degree to which people paid attention to targets of each status level, by taking the mean across the high, medium, and low-status targets in the four videos. The mean (and standard deviation) peer-ratings for each type of target (on our 7-point scale) were 5.78 (0.6), 4.99 (0.2) and 3.25 (1.5) for high, medium and low status respectively. These ratings were significantly different $(F(2,9)=7.7$, $p<.05)$. Planned contrasts across the in-group ratings of the four examplars of each target (i.e., across the four 'high-status' targets, the four 'low-status' targets, and the four 'medium-status' targets) confirmed that low-status targets were rated significantly lower in status than high $(t(9)=3.83, p<.01)$ and medium $(t(9)=2.63, p<.05)$ status targets. High and medium targets did not differ significantly. As a further manipulation check, therefore, we asked our eye-tracked participants (after the experiment), and an additional 34 naïve raters, to rate the social status of each of the targets (using the same three items and 7point scale as the in-group ratings). These participants rated our three groups of targets as significantly different $\left(F(2,174)=110.46, p<.001, \eta_{p}^{2}=.56\right)$. High-status targets $(M=4.97, \mathrm{SD}=1.20)$ were rated higher than medium-status targets $(M=4.21, \mathrm{SD}=1.06)$, who in turn were perceived as having significantly higher status than lowstatus targets $(M=2.25, \mathrm{SD}=0.76)$. Contrasts between these levels were all highly reliable (all $t s(174)>4$, ps $<.001$ )

In this experiment, we were particularly interested in whether social status made a difference to an observer's
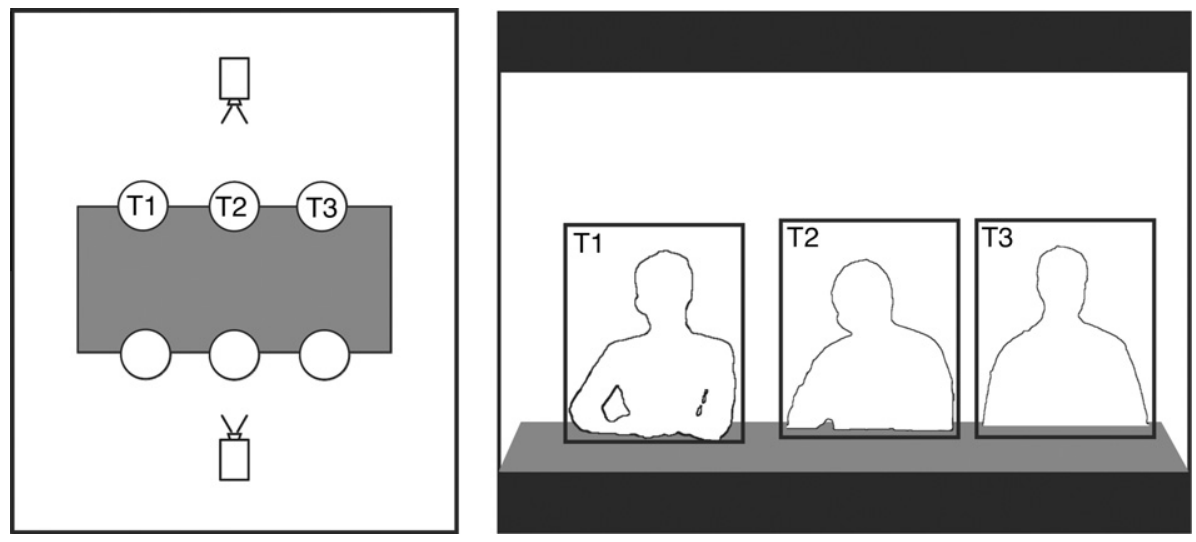

Fig. 1. Stimuli production and layout. The videos were filmed using a camera facing each side of a table and capturing three of the people in the group (e.g., targets T1-T3; left panel). Frames from the clip featured these three people sitting side by side at a table (right panel, illustrating approximate size of the targets). The ROIs used to define fixations on the targets are shown as black boxes. 
gaze allocation, even when the observer had only brief exposure to the target individuals. Given the difficulty of analyzing eye movements in video, and of maintaining an accurate track over long periods of time, we used six twenty-second clips for each set of targets. One research assistant blind to the study's hypotheses was instructed to choose six clips from each video that featured group members negotiating and reaching a pivotal decision. These clips often contained moments of conflict, or times when one target had succeeded in persuading other members after an extensive debate, thus they captured moments where status dynamics were particularly salient.

The clips were cropped and formatted as digital movie files with dimensions of 1024 by 768 pixels and a frame rate of $30 \mathrm{fps}$. The aspect ratio of the original clips was $16: 9$, and thus a black border was added above and below the video image. The Xvid video codec (www.xvid.org) was used as it offered superior playback, as well as extremely accurate timing, which meant that the eye tracking apparatus could log exactly which frame was on the screen at any one time. Sound was played via an ASIO sound card, which maintained synchrony between video and audio. Each participant saw all six clips from one set in a random order. The set of clips seen by each participant was determined randomly, and each set of clips was seen by six participants, with the exception of one set that was seen by seven participants.

\subsection{Apparatus}

The videos were shown on a 19-in. colour monitor with a refresh rate of $60 \mathrm{~Hz}$. Participants used a headrest, which minimized head movements and ensured a constant viewing distance of $60 \mathrm{~cm}$, which resulted in an effective screen size of $40^{\circ}$ by $31^{\circ}$ of visual angle. At this distance, the visible area of the video frame was approximately $40^{\circ}$ by $23^{\circ}$. Sound was played through a pair of speakers positioned on either side of the monitor.

Eye movements were recorded using the EyeLink II system, which uses a head mounted camera. Pupil position was recorded monocularly from the video image of the right eye at $500 \mathrm{~Hz}$. The EyeLink system used an on-line parser to extract fixations and saccades from the eye position samples, using velocity $(30 \% \mathrm{~s})$ and acceleration $\left(8000^{\circ} / \mathrm{s}^{2}\right)$ thresholds.

The dynamic nature of our stimuli meant that maintaining a temporal synchrony between video, audio and eye tracking data was important. We accomplished this using EyeLink's standalone Experiment Builder software, which, in concert with the Xvid codec, wrote with millisecond accuracy time-stamped messages to the eye tracking data file at the onset (i.e. the first screen retrace) of each video frame. Several frames were cached in advance and the system demonstrated extremely accurate timing. The timestamp for each frame could then be compared to the events in the eye tracking data file (e.g. fixations).

\subsection{Procedure}

The experiment began with the instruction that the participant should watch the clips as if they were in the room with the targets. More specifically, they were instructed to "imagine that you're in the room with these people, working on the task. Please think about which of the people in the group you would want to work with in a subsequent task". The sound volume was adjusted for each participant, and the eye tracker was calibrated with a 9-dot calibration routine that presented dots one at a time in known locations on the screen.

The trials then began. In each of the six trials, a driftcorrect marker was first presented in the centre of the screen, and participants were required to look at the dot and press a key on the keyboard when central fixation was attained. This had the effect of constraining the initial fixation position to the centre of the screen, and correcting the eye tracker for any eye drift. The clip then appeared and the video and audio were played at normal speed for their duration of $20 \mathrm{~s}$. Eye movements during this time were recorded, along with a record of timestamps indicating the onset time of each frame of the video. All six trials proceeded in this fashion.

\section{Analysis and results}

\subsection{General viewing behaviour}

We first assessed how participants responded to the clips by looking at the general eye movements they made.

Participants made an average of 49 fixations $(S D=8.4)$ during each 20s clip, with fixations having a mean duration of $377 \mathrm{~ms}(\mathrm{SD}=83)$. The saccades between these fixations had a mean amplitude of $6.6^{\circ}(\mathrm{SD}=1.2)$. In all subsequent analysis, the fixation at clip onset was not included, because its central position was constrained by the procedure preceding the onset of the clip. To move beyond these simple descriptives, we quantified the attention given to the three people in the clip (i.e., the "targets") by defining a region of interest (ROI) around each person. This region was a rectangle with dimensions $10.9^{\circ}$ by $14.1^{\circ}$, a size that was kept constant for all targets. In most cases, there was relatively little movement of the targets within a clip, but for this first analysis the ROIs were large enough to encompass the targets throughout the whole clip. The ROIs for one clip are depicted in Fig. 1 (right). Using these ROIs, we classified fixations as landing on one of the targets or on the background of walls, furniture and blank screen.

Across all clips and observers, an average of $77 \%$ of all fixations landed on the targets. It was relatively rare for the observers to look at the empty and static furniture and background. The ROIs covered $37 \%$ of the screen area, so if fixations were uniformly distributed we should expect approximately this proportion of fixations to land on the targets. The fact that many more fixations were spent looking at the targets in the clips than this mean chance expectancy is preliminary evidence that participants focused their attention on the targets. A possible problem with this interpretation is that fixation distributions in a range of stimuli tend to be highly centralized (Foulsham \& Underwood, 2008). As one of our ROIs was central, close to where viewing began and where participants tend to fixate, it might be that this underlies the tendency to fixate the tar- 
gets. However, this explanation is unlikely to account for the data: peripheral targets were also fixated much more often than we would expect given their area, despite being further from the centre of the screen (44\% of fixations landed on the left or right target, which together covered just $25 \%$ of the screen area). Thus, the people in the clips were potent at attracting fixation. Our subsequent analyses examined whether this varied as a function of these targets' social status.

\subsection{Gaze allocation and social status}

Each clip had three targets, classified as high, medium or low social status. We analysed the eye movement data using repeated measures ANOVA with one within-subject factor of social status. Table 1 shows the measures taken for each level. First we considered the proportion of fixations that landed on the different targets. Fixations were parsed by the EyeLink system, according to the velocity and acceleration thresholds outlined in the method, and proportions were calculated across all fixations made during a clip, and then averaged across clips.

Status had a reliable effect on the proportion of fixations on the target $\left(F(2,48)=31.7, p<.001, \eta_{p}^{2}=.57\right)$. There were more fixations on high-status targets than on medium-status targets, who received more fixations that low-status targets (all planned comparisons $p<.001$ ). This difference was quite pronounced. For example, mediumstatus targets received twice as many fixations as low-status targets, and high-status targets received even more attention.

An alternative way to measure the amount of attention paid to the different individuals in a clip is to look at the fixation time committed to each target. This was defined as the sum duration of all the fixations on each target, and it was averaged across the six clips to give the total fixation time per 20s clip. This measure reflects differences in how long observers looked at one target on each occasion, over and above the number of fixations. As previously, there was an effect of social status $(F(2,48)=34.1$, $\left.p<.001, \eta_{p}^{2}=.59\right)$. Pairwise comparisons showed the same pattern as the previous analysis: observers spent the most time looking at the high-status target, followed by the medium-status target, with the low-status target being inspected for the least amount of time (all $p<.01$ ).

The measures so far demonstrate that social status had an effect on the amount of attention given to the people in the clips. These measures were taken across a whole 20s

\section{Table 1}

Means (with standard errors in parentheses) for the different measures taken, as a function of social status.

\begin{tabular}{llll}
\hline & \multicolumn{3}{l}{ Target social status } \\
\cline { 2 - 4 } & High & Medium & Low \\
\hline Mean proportion of fixations & 0.35 & 0.28 & 0.14 \\
& $(0.02)$ & $(0.01)$ & $(0.01)$ \\
Total fixation duration per clip (s) & 6.47 & 4.86 & 2.30 \\
& $(0.47)$ & $(0.33)$ & $(0.18)$ \\
Mean gaze duration (ms) & 994 & 767 & 669 \\
& $(74)$ & $(68)$ & $(45)$ \\
\hline
\end{tabular}

video, comprising 10-20 fixations with a total duration of several seconds. An alternative question concerns how long the targets were gazed at on each visit, before a different person was inspected. For example, it is possible that high-status individuals are looked at more often, and also that they hold an observer's attention for longer on each occasion that they are looked at. To explore this, we measured the mean gaze duration. A gaze was defined as the sum duration of all consecutive fixations on a target, with each gaze ending with a shift to a new target or to the background. On average, gazes were $810 \mathrm{~ms}$, which corresponds to 2 or 3 fixations before shifting to a different region. Mean gaze duration was affected by social status $\left(F(2,48)=12.9, p<001, \eta_{p}^{2}=.35\right)$. The average length of each gaze on the high-status person was reliably longer than that on either of the other targets $(p<.05)$. The lowstatus person received the briefest gazes, although the comparison between medium and low status fell short of significance.

Although the effects of social status on fixation behaviour are interesting, it is important to rule out more basic factors. One such factor is the spatial position of the people in the clips. As previously mentioned, people tend to fixate close to the centre of an image or video, and although seating was assigned to targets on a random basis, those seated in the centre may have taken on a high-status role as a result of their position. In fact, the low-status target was never positioned in the centre. Thus, centrality could explain the attentional bias away from these targets. However, in three of the four groups the high-status target was positioned on one side or the other, making centrality unlikely to explain the advantage for high-status over medium-status individuals. To further explore this issue, we conducted an additional analysis, comparing mediumand high-status targets in different positions. For this analysis, and for all those that follow, we focused on the proportion of fixations allocated to the different types of target, as the results from this measure and that of total fixation duration were identical. Fig. 2 shows the proportion of fixations for the different types of target, both when they were positioned in the centre, and when they were positioned at the sides. It is clear from the graph that, although central targets were more likely to be fixated, the effect of social status was very similar at both spatial positions. High-status targets received more fixations on average than medium-status targets, both when they were each on the side of the display, and when they were both in the centre (both $t(23)>2.6, p<.02$ ). This is good evidence that the effect of status is not just an artifact of spatial position. Data for the low-status individual in the centre was not available because this target was positioned at the side in all clips, but given the results for high and medium-status targets, the low-status targets' position is unlikely to have substantially influenced our results. To summarize this analysis, although seating position did matter (presumably because of a bias for fixations on the centre of the display), social status had an impact on attention at all seating positions.

One question raised by these results is whether the effect of social status on eye gaze is spontaneous, or partially due to our instructions to eye-tracked participants, to 


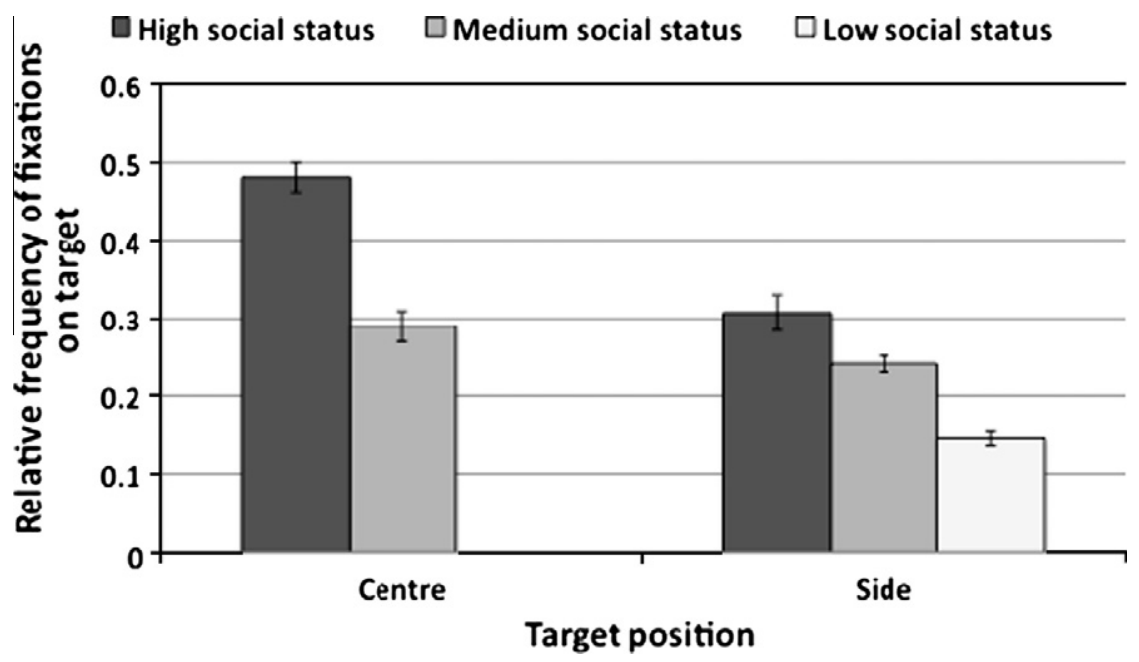

Fig. 2. The proportion of fixations on targets that appeared at the centre or the sides of the group. Bars show the mean with standard error bars.

\begin{abstract}
"think about who you would want to work with on a subsequent task"; it is possible that these instructions encouraged observers to look at the high-status individuals. Although this issue does not change our main finding, that individuals can automatically orient their gaze toward high-status individuals and determine which individuals in a group interaction are high-status, despite viewing these individuals for only very brief time periods, we conducted a control experiment to determine whether this process is spontaneous or potentially goal-directed. We tested an additional seven participants (with the same characteristics as those in the main study) as they watched one of the four sets of clips, and we instructed these observers to "think about who you would NOT want to work with (i.e., who you would want to avoid working with) on a subsequent task". All other parts of the experiment were unchanged. The results showed that there was still an effect of social status on the proportion of fixations $\left(F(2,12)=4.8, p<.05, \eta_{p}^{2}=.45\right)$ and the total fixation time $\left(F(2,12)=6.2, p<.05, \eta_{p}^{2}=.51\right)$, and that in each case the high-social status target was prioritized over the medium target, who was in turn gazed at more than the low target (all $p s<.05$ ). Thus, even when instructed to think about the least effective group member, observers spontaneously selected targets according to social status. These findings indicate that prioritizing high-status individuals with eye gaze is a spontaneous rather than induced behaviour.
\end{abstract}

\subsection{Gaze allocation and speaking}

The eye movements of observers were sensitive to social status, and it is interesting to demonstrate this with complex stimuli and over only a short clip. What target behaviours underlie this effect? A strong candidate is the verbalizations of the individual. If high-status targets do most of the talking, and observers tend to look at the person speaking, this would explain our previous results. This would not be a trivial result, but it is important to ask whether status might have an effect in addition to that moderated by verbalizations.

Our eye movement methodology allowed us to look at the distribution of attention over time, with a high temporal resolution. To investigate how this distribution was related to verbalizations, we compared the fixation data to a record of who was talking at each moment in the clips. This record came from a trained independent observer, who watched all the clips and logged the beginning and end of each utterance. Specifically, we used custom-designed software that played the clips at a slow speed and allowed the observer to press one of two keys to indicate that a target had started or finished talking. This was repeated three times per clip (once for each target), and the result was a frame-by-frame timing matrix that showed which people, if any, were talking at any time (see Fig. 3, top). As one might expect, the amount of time a target spent talking was related to their social status (one-way ANOVA across clips, $\left.F(2,71)=11.4, p<.001, \eta_{p}^{2}=.25\right)$. High-status individuals spent the greatest proportion of the clips talking, followed by the medium-status targets and the low-status targets (means across all of the clips $=26 \%, 19 \%$ and $5 \%$ respectively). Pairwise comparisons demonstrated that the low-status targets spoke for reliably less time per clip than either the high-status or the medium-status targets (both $p<.005$ ). The difference between high-status and medium-status targets was not significant $(t(46)=1.3$, two-tailed $p=0.19$ ). Importantly, the absence of a significant difference in speaking time between high- and medium-status targets suggests that the reported attentional differences between these targets cannot be solely explained by speaking time.

To control for both position and speaking time directly, we ran an analysis by target, comparing the average proportion of fixations that each target received in each clip but adding target position (centre or side) and the proportion of time this target spent talking (in this clip) as covariates. This ANCOVA procedure statistically adjusted the dependant variable (mean proportion of fixations) to par- 


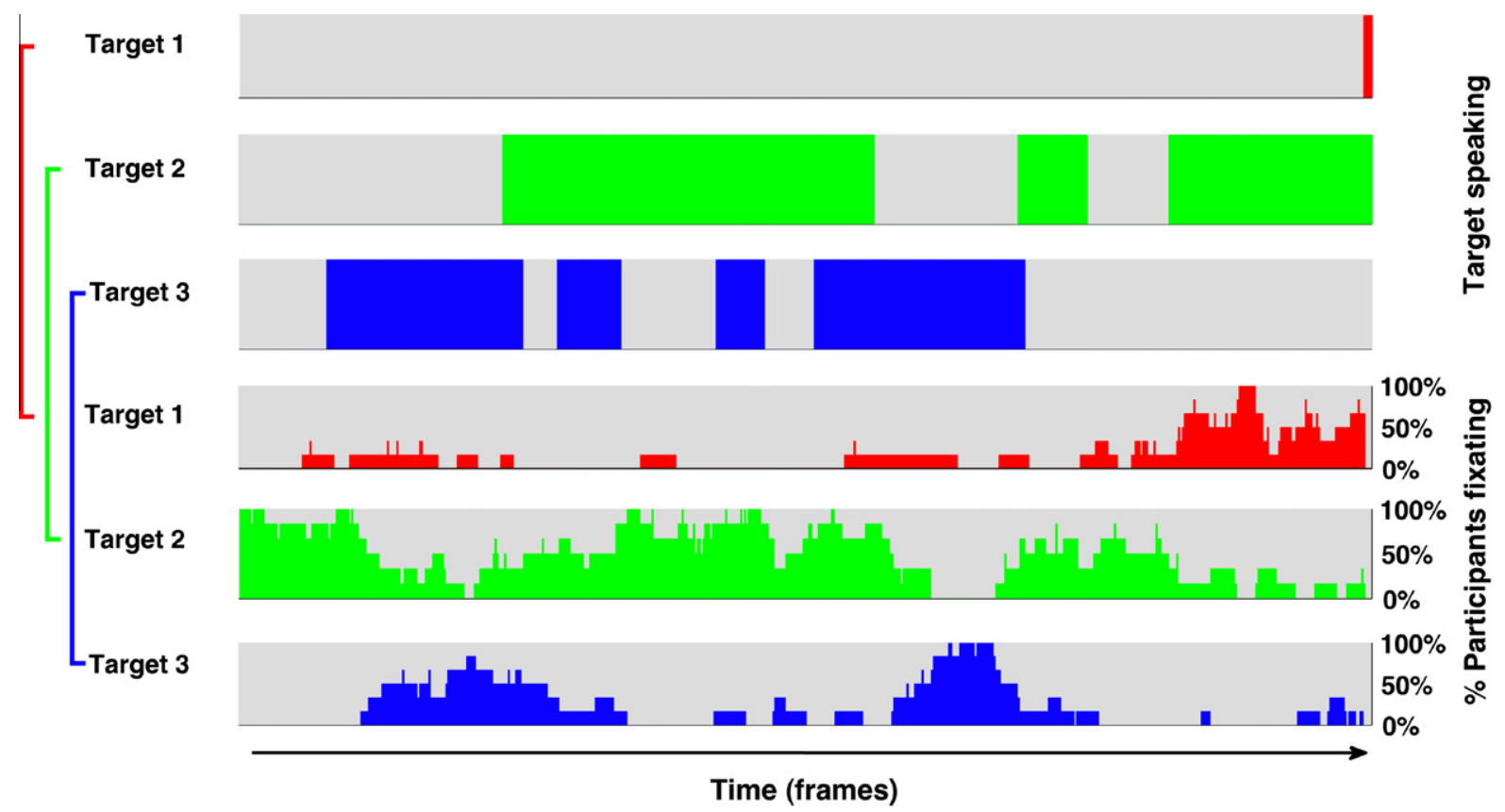

Fig. 3. The synchrony between gaze and talking for one example clip, with time along the $x$-axis for a duration of 20 s. The top three panels show whether each of the three targets in the scene (numbered 1-3 from left to right) was speaking at each point in time, with a solid bar indicating that they were. The bottom three bars show the proportion of observers watching the clip who fixated each of these people over the same time course. In many cases, a peak in participants looking at an individual coincides with that individual talking. In this clip, target 1 was low social status, target 2 was high-social status and target 3 was medium-social status.

tial out the effects of speaking time and position. As expected from our previous analyses, both seating position $\left(F(1,67)=12.3, \quad p<.005, \quad \eta_{p}^{2}=.15\right)$ and talking time $\left(F(1,67)=35.9, p<.001, \eta_{p}^{2}=.35\right)$ influenced the attention given to each target. Targets who sat in the centre and spent more time talking were fixated most often. Most important, however, social status continued to have a reliable effect on the allocation of fixations over and above that predicted by the seating position and speaking time of the target $\left(F(2,67)=16.8, p<.001, \eta_{p}^{2}=.33\right)$. The same hierarchy of attention was seen, with high-status targets being fixated more often than medium-status targets and low-status targets receiving the fewest fixations (all pairwise comparisons $p<.05$ ).

Fig. 3 shows a graphical representation of one of the clips from the experiment. This visualization compares the record of who was speaking at any point in the clip (top three panels), to the proportion of observers who were fixating each target at that time (bottom three panels). At several points in this figure there is a tendency for observers to fixate the person who is talking. To explore this further we categorized all the fixations in a clip according to who was talking in the frame at which the fixation started. At this point we were interested in how the destination for each fixation was planned or guided, so we categorized fixations with regard to their start time, as this will reflect the aspects of the target that attracted gaze toward them, rather than changes that occurred while the observer was fixating. We analysed all fixations, and made a comparison between the proportion of these fixations that were directed at the person talking (at the start of that fix- ation) and those that were on another target or on the scene background. Table 2 summarizes the relationship between who was speaking and who was being fixated.

As found in previous analyses, in general, when a target was talking participants were most likely to look at that person, and this can be seen in the relatively high values along the diagonal in Table 2. Did this trend vary according to the status of the person speaking? When the high-status person was talking, target status had a reliable effect $\left(F(2,48)=74.1, p<.001, \eta_{p}^{2}=.75\right)$. In this case the highstatus speaker was fixated on almost half of all fixations, but on those occasions when someone else was fixated while the high-status person was talking, it was more likely to be the medium-status target than the low-status individual (all levels different at $p<.001$ ).

The targets also received different amounts of attention when the medium-status target was speaking $\left(F(2,48)=43.3, p<.001, \eta_{p}^{2}=.64\right)$, with the person who was talking (in this case the medium-status target) again receiving the most fixations. However, when fixations were not on the medium-status target, the high-status target was more likely to be fixated than the low-status target $(p<.01)$, even though neither of these targets were speaking. The low-status person was the least potent at attracting fixations when he/she was talking, and on these occasions participants were almost as likely to look at the high-status target as the speaker. There was no effect of status when the low-status target was talking $\left(F(2,48)<1, \eta_{p}^{2}=.02\right)$ and none of the pairwise comparisons were different. The clearest demonstration that the effect of social status on gaze can be dissociated from 
Table 2

The relative frequency of fixations on each type of target, and on the background, expressed as a proportion of the total made while each target was speaking. Each cell shows the mean across participants, taking into account the differences in how often each target spoke. The first column, for example, shows who was fixated during the time that the high-status target was speaking.

\begin{tabular}{|c|c|c|c|c|c|}
\hline & & \multicolumn{4}{|c|}{ Target speaking } \\
\hline & & High-status & Medium-status & Low-status & Nobody \\
\hline \multirow{5}{*}{ Target being fixated } & High-status & $49 \%$ & $22 \%$ & $30 \%$ & $34 \%$ \\
\hline & Medium-status & $22 \%$ & $47 \%$ & $23 \%$ & $26 \%$ \\
\hline & Low-status & $11 \%$ & $11 \%$ & $31 \%$ & $16 \%$ \\
\hline & Background & $18 \%$ & $20 \%$ & $16 \%$ & $24 \%$ \\
\hline & Total & $100 \%$ & $100 \%$ & $100 \%$ & $100 \%$ \\
\hline
\end{tabular}

speaking is apparent from the pattern of results on occasions when nobody was speaking: looking only at these fixations, there was an effect of social status $\left(F(2,48)=16.3, p<.001, \eta_{p}^{2}=.41\right)$, showing precisely the same pattern as observed previously: the high-status target was fixated more than the medium-status target, with the low-status target receiving the least attention (all comparisons $p<.05$ ). Thus, although people tended to look at the person speaking, social status remained important even when nobody was talking.

An alternative way of analyzing the fit between gaze and speaking is to use cross correlation. This technique analyses the correlation between two signals over time, and it provides a correlation coefficient when the two signals are perfectly aligned (the "zero lag"), as well as when one signal is shifted relative to the other (see Richardson \& Dale, 2005, for a similar approach). In our case, we computed a cross correlation for each target, in each clip, between the record of speaking and the proportion of observers watching that clip who were fixating that target. We can then ask: (a) whether this correlation over time is statistically different from zero, and (b) whether the highest correlation occurs at the zero lag. If the highest correlation were found at a different lag, it would suggest that there was a temporal delay between gaze and speaking. For example, observers might have looked at individuals a few frames after they started speaking. To give an estimate of the correlation we would expect by chance, we also made two sets of control comparisons. First, we compared the fixation record from each target and clip to the speaking record for all other targets and clips, which gives a baseline similarity between any two random gaze and speaking signals. Second, we compared the gaze data from each target and clip with the speaking record of the same target in each of the five other clips in which that target appeared. This "matched target" comparison gives a measure of the chance correlation expected between fixations on a person and the speech of that same person in other situations. Table 3 shows the results of these analyses.

Several interesting points can be drawn from this analysis. First, the cross correlation between a person speaking and their being fixated was reliably greater than zero. In comparison, the control data sets of fixations matched to the speaking data from other clips produced no correlations at the zero lag and much smaller correlations when maximally aligned. Second, this correlation was higher still if we assume that there is a temporal offset in the relationship between speaking and fixation. The average lag at

\section{Table 3}

Summary statistics from a cross correlation analysis of speaking and fixation. Cells show the mean (and standard error) correlation across all clips and targets.

\begin{tabular}{|c|c|c|c|}
\hline & $\begin{array}{l}\text { Observed } \\
\text { data }\end{array}$ & $\begin{array}{l}\text { Random } \\
\text { control } \\
\text { data }\end{array}$ & $\begin{array}{l}\text { Matched } \\
\text { target } \\
\text { control data }\end{array}$ \\
\hline Cross correlation at zero lag & $\begin{array}{c}0.38 \\
(0.03)\end{array}$ & $\begin{array}{l}-0.01 \\
(0.005)\end{array}$ & $\begin{array}{c}-0.01 \\
(0.01)\end{array}$ \\
\hline Maximum cross correlation & $\begin{array}{c}0.45 \\
(0.03)\end{array}$ & $\begin{array}{c}0.12 \\
(0.004)\end{array}$ & $\begin{array}{c}0.13 \\
(0.01)\end{array}$ \\
\hline
\end{tabular}

which the highest correlation was found can show the direction of this offset. Across all comparisons, the median lag at which the highest correlation between speech and fixation was found was -5 frames. Surprisingly, the negative offset indicates that, on average, correlations were higher when fixations were compared with the speaking that was going to take place five frames in the future. In other words, people tended to look at the speaker slightly ( $\sim 150 \mathrm{~ms}$ ) before they started talking. The pattern for gaze to precede speech was found across targets of different status, although the extent of the offset differed slightly (median lags for high, medium and low status $=3,5$ and 7 frames, respectively). This variability would be interesting to study further, but, because it was not the focus of the present study, we included a relatively small number of clips and targets of each level of status and relatively few frames where low-status targets were talking, so this potential status difference should be interpreted with caution.

\subsection{Regions of interest analysis}

The previous analyses were based on relatively large areas of interest covering the whole of each person within the clip, and they showed that the targets were very frequently inspected. Which part of these targets was most potent at drawing gazes? There is a large literature showing the importance of faces, and in particular eyes, in drawing attention (Kingstone, 2009). In static photographs, people often spend most of their time looking at the faces and eyes of the people in the scene (Birmingham et al., 2008). We therefore looked to see if the same was true in our dynamic movie clips, and also if this varied with social status. Because our targets would have moved slightly over the 20s clips, we first needed to define moving regions of 


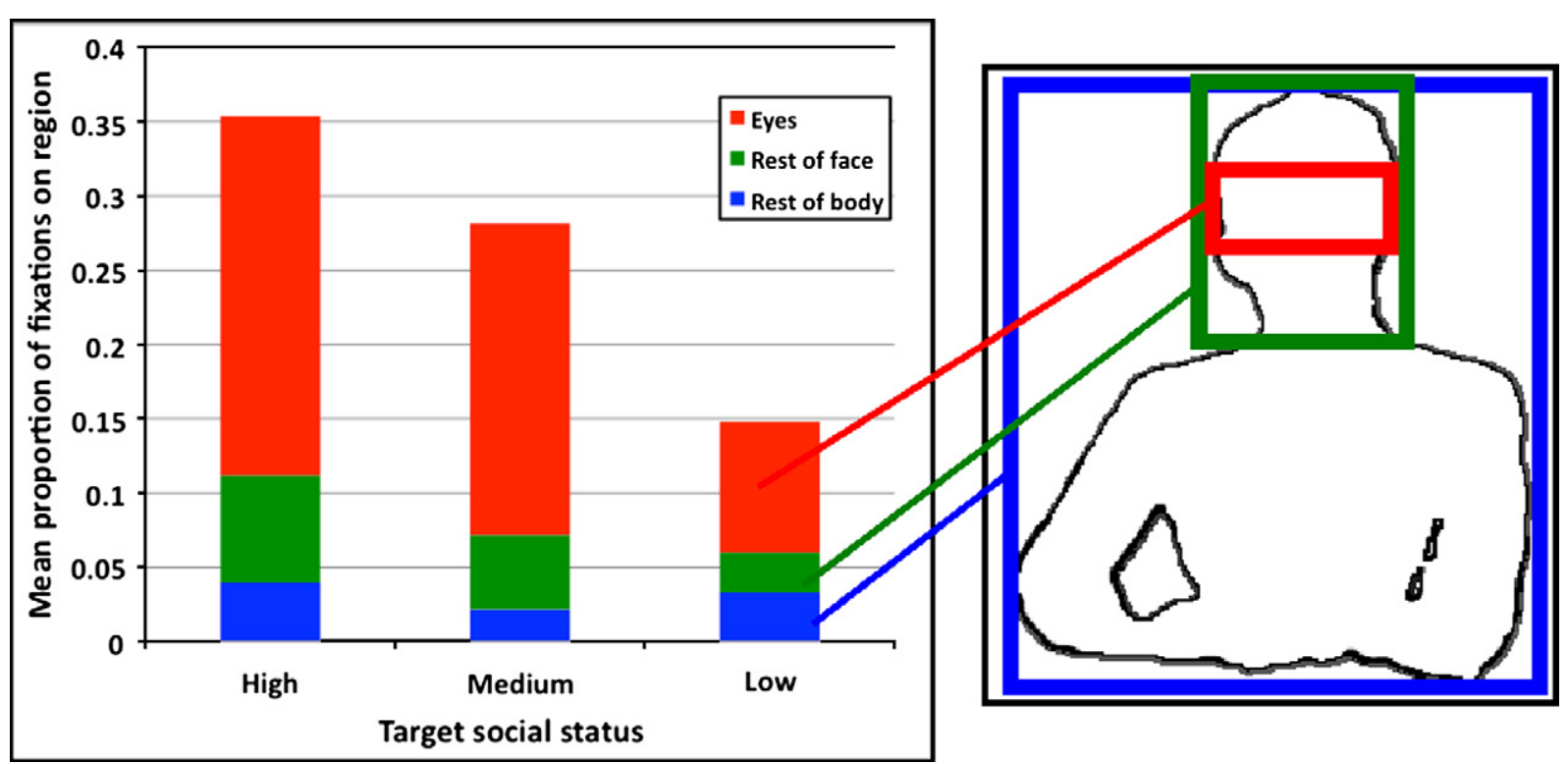

Fig. 4. Measuring the amount of gaze given to different parts of the people was accomplished by defining moving areas of interest for the eyes and head (the relative sizes of which are depicted with a diagram of one target in the right panel). The proportion of fixations on each of these regions, averaged across all observers, is shown in the left panel.

interest. This was done by hand using custom software in MATLAB. Each clip was played at a slow speed, and a mouse cursor was moved to follow the region in question, resulting in a record of where that region was at any frame in the movie. We did this for both the head region (which was kept to a standard size of $3.9^{\circ}$ by $5.8^{\circ}$ ) and the eye region $\left(3.9^{\circ}\right.$ by $\left.1.9^{\circ}\right)$ and for each target person. Fixations could then be labeled according to their location in the frame at which the fixation started. For example, a fixation was classified as on the eyes if, on the frame where it started, its spatial coordinates lay within the eye region. Fig. 4 shows an example of these regions, and the relative frequency of fixations on the eyes, the rest of the head (defined as head minus eyes) and the rest of the body (defined as the original target ROIs minus the head).

We analysed the proportion of fixations on each region using repeated measures ANOVA with two factors: social status (high, medium, or low) and region of interest (eyes, rest of head, or rest of body). As previously, there was a significant effect of status $\left(F(2,48)=31.8, p<.001, \eta_{p}^{2}=.57\right)$. There was also a main effect of region of interest $\left(F(2,48)=74.7, p<.001, \eta_{p}^{2}=.78\right)$. Summing across all targets, the mean probability of a fixation landing on someone's eyes was $54 \%$, much greater than gazes to the rest of the face $(15 \%)$ or to the body (10\%). All these averages were reliably different (all ps $<.05$ ). There was also a reliable interaction $\left(F(4,96)=22.5, p<.001, \eta_{p}^{2}=.48\right)$, showing that the potency of the different regions at drawing fixations varied with the social status of the target. Looking at the simple main effects of region of interest at different levels of status, the trend for the eyes to be most frequently fixated followed by the face and then the body was the same in both high- $\left(F(2,23)=52.2, p<.001, \eta_{p}^{2}=.82\right)$ and medium-status targets $\left(F(2,23)=77.3, p<.001, \eta_{p}^{2}=.87\right)$. In each case, comparisons between the different regions of interest were all reliable (at least $p<.05$ ). There was also an effect of region of interest in the low social status target $\left(F(2,48)=31.7, p<.001, \eta_{p}^{2}=.73\right)$. In these targets, there was still a tendency to fixate the eyes rather than the face or body (both $p<.001$ ). However, unlike in the other targets, there was no reliable difference between the likelihood of looking at the face compared to the body.

\section{Discussion}

This experiment explored the spatiotemporal distribution of gaze in a controlled but realistic video of a social interaction. Unlike the vast majority of research into social attention, we used stimuli containing several individuals conversing in a dynamic situation (a video), and this allows us to draw some conclusions about how visual attention is directed in complex scenes with a truly social element. The evolutionary research reviewed in the introduction leads to the straightforward predictions that humans should be predisposed to attend to other people in the environment, to their eyes (Emery, 2000), and to high-status people in particular (Henrich \& Gil-White, 2001). Testing these predictions led to several interesting findings.

First, people chose to spend a majority of the time looking at the people in the clips, even though these people did not occupy the entire scene. Of course this is not all that surprising considering that the other regions in the movie (background and furniture) were motionless, not useful for the task, and probably not as salient in terms of low level features, but it does confirm previous reports that the visual attention system is particularly inclined to select people, and extends these findings to video. More interesting, most of the fixations on people were targeted at an individual's eye region, with fewer gazes directed at the rest of the 
face, and fewer still at the torso and other body parts. Participants spontaneously chose to monitor the eyes of the people in the clips, and this extends to natural dynamic scenes what has previously only been found for static images (Birmingham et al., 2008) and Hollywood movies (Klin et al., 2002).

Humans have physiologically evolved to communicate their eye gaze direction to others (for example by having a high contrast between the iris and sclera see Kobayashi \& Koshima, 1997), but the function of attentional orienting to the eyes is not fully understood. Evidence suggests, however, that the ability to share others' goals and intentions (i.e., theory of mind) is fundamentally linked to orienting to another's gaze (Tomasello, Carpenter, Call, Behne, \& Moll, 2005). For example, evidence from individuals with autism (Baron-Cohen, 1995), psychopathy (Dadds, El Masry, Wimalaweera, \& Guastella, 2008), and patients with selective damage to the amygdala (Adolphs et al., 2005), confirms that deficits in processing emotion and theory of mind are often accompanied by a reduced tendency to look at the eyes. The hormone oxytocin enhances both social approach behaviour and fixations to the eyes in humans, confirming this link and suggesting part of the mechanism (Guastella, Mitchell, \& Dadds, 2008). In our naturalistic task, watching a social interaction while thinking about some of the people involved, the eyes were spontaneously selected by observers as being especially useful. This is consistent with a theoretical model that attentional processing of the eyes functions to enhance the perception of the target's external goals (e.g. who they are talking to) as well as their internal emotions, intentions and beliefs (e.g. how they feel about that person; see Birmingham \& Kingstone, 2009 for a recent review)

Second, a range of different measures demonstrated that the relative social status of the people in the clips had a large and robust effect on who was fixated. People who were previously rated as having high social statuswhom other group members perceived as having led the task or influenced the group-were fixated more often, for longer on each gaze, and for a longer total time, compared to people seen as medium social status, or low social status, and low-status targets received the least attention. The independently rated status hierarchy of the group depicted in the videos had a highly systematic effect on the distribution of gaze of participants watching the clips. Why did social status affect how much a person was looked at? Although both the position of a person in the scene and their verbalizations had an effect on the amount of attention they received, our analyses indicate that the effect of social status could not be attributed to either of these factors. This was clear in multiple different analyses. For example, high-status people were looked at more often than medium-status people whether they were positioned in the centre of the group or on the sides. High-status targets spoke slightly more often than medium-status targets (although this difference was not statistically significant), but the effect of the social status hierarchy on attention held even in those moments when nobody (or somebody else) was talking, and when variance in speaking time was statistically removed. Our eye-tracking methodology allowed us to look in detail at the gazes that each target received, and the three measures reported can reveal slightly different aspects of the bias shown towards high-status targets. The fact that participants spent a greater amount of total time looking at these targets-often several seconds more within a short 20s clip-could be attributed to a higher frequency of shifts toward these people or a longer time spent looking at them each time they were there. In fact, both these patterns were found, with participants making more fixations on high-status targets as well as longer gazes. These findings demonstrate that people are more likely to shift their gaze to high-status targets, and that once there they stay there for longer before looking at someone else. Our control study demonstrated that the selection of high-status individuals occurred even when we asked observers to think about people that they would not want to work with, demonstrating that the effect of status on gaze direction was not specific to our instructions, but rather represents a spontaneously adopted pattern.

The strong effects of social status are particularly interesting given that participants saw only brief episodes of the social interaction in each group. One explanation of the high-status advantage is that status was inferred from aspects of the targets' appearance, from their non-verbal behaviour, and from other group members' behaviours and responses toward them (e.g., others asked them for advice, deferred to their opinions, etc.), which jointly determine a target's position in the social hierarchy. In particular, status rank differentiation may be the result of individual differences in dominance (i.e., an individual's ability to be forceful and intimidate others) and prestige (i.e., an individual's ability to demonstrate valued skills and expertise; Henrich \& Gil-White, 2001). Indeed, in the group task described here, individuals who were perceived to be prestigious or dominant by other group members attained the highest level of overall status and influence over others (Cheng et al., in preparation). Further research is necessary to identify whether specific behaviours (e.g., elements of non-verbal or verbal behaviour) are in themselves salient attractors of attention, and to address the question of whether a conscious attribution of status is necessary for the effect on attention. In the case of speaking, our findings demonstrate that high-status targets were looked at more often than medium- and low-status targets even when somebody else, or nobody, was talking, suggesting that it is their status within the hierarchy, rather than their verbal behaviour at that time, that results in them being paid the most attention.

Regardless of these issues, the present findings suggest that observers can very quickly ascertain who the high-status members of a group are, and are predisposed to orient toward these people. This attentional bias may represent an evolved cognitive mechanism that facilitates the detecting and monitoring of high-status individuals (Cheng et al., in preparation; Henrich \& Gil-White, 2001). Increased attention toward these individuals might allow group members to appropriately monitor the goals and behaviours of their leaders, learn from these individuals, who tend to possess superior skills (i.e., if they demonstrate prestige), and monitor potential threats or attacks from 
these more powerful conspecifics (i.e., those who demonstrate dominance). Converging lines of research show that young children (3-4 year olds) automatically track the gaze of other people, and preferentially imitate the preferences of those most gazed at by others, even when they are alone (Chudek, Heller, Birch, \& Henrich, submitted for publication). These data also lend strong support to the idea that others could use gaze following as an indicator of social status within a group: the person who receives the most glances from other group members, or who is gazed at the longest may be perceived as the high status individual (Chance, 1967; Emery, 2000).

Given that we interpret the differences in eye movements as evidence for sensitivity to the social context, and particularly the status hierarchy, it is likely that these effects will be moderated by individual differences known to influence this sensitivity. For example, observers who are themselves considered low social status should be more inclined to look at high-status individuals, so as to monitor and learn from their superiors (in the same sense that monkeys only follow the gaze of conspecifics who are of higher social status; Shepherd et al., 2006). In fact, previous research suggests that observers low in feelings of belongingness show heightened gaze-following tendencies (Wilkowski, Robinson, \& Friesen, 2009). The gender of observers and targets may also make a difference as women and men prioritize different attributes in potential mates (Li, Bailey, Kenrick, \& Linsenmeier, 2002). Such predictions are fuel for further research, and the paradigm used here may prove fruitful for testing them.

Another factor that might affect results is targets' relative attractiveness. Attractive people are thought to draw attention (Maner et al., 2003), and attractiveness has been found to predict high social status in some groups (Anderson, John, Keltner, \& Kring, 2001). The present study design may provide a suitable context for testing the effects of attractiveness on attention in dynamic contexts, particularly as there is evidence that attractiveness inferred from watching dynamic video might prove to be different from image-based, "physical” attractiveness (Riggio, Widamen, Tucker, \& Salinas, 1991). While we did not obtain ratings of attractiveness in the present study, we did examine the related construct of interpersonal liking, and found that: (a) the effects of social status on attention remained significant even after controlling for ratings of peer liking, and (b) although liking had a small positive effect on attention, this effect was reduced to non-significance once speaking time was accounted for. This apparent null association between liking and attention leads to the speculation that physical attractiveness is not likely to completely explain the observed attentional biases, which clearly point to a status advantage. Nonetheless, disentangling these influences on attention would be a fruitful avenue for further research. However, it is important to bear in mind that status and attractiveness may be too interdependent to fully tease apart these relations; given evidence that attractiveness partly determines status, controlling for this variable may be a case of "throwing out the baby with the bathwater".

By examining the temporal synchrony between who was speaking and who was being looked at, our experi- ment also addressed the relationship between gaze and speech. A significant body of research has investigated where people fixate when observing someone talking. Somewhat surprisingly, in general both humans (Vatikiotis-Bateson, Eigsti, Yano, \& Munhall, 1998) and monkeys (Ghazanfar, Nielsen, \& Logothetis, 2006) look mostly at the eye region, rather than the mouth region, of a vocalizing conspecific. This pattern is confirmed in our finding that the eyes were indeed looked at most frequently. When auditory noise is added to the speech, or when the task requires accurate auditory discriminations, a higher frequency of fixations are made to the mouth (Buchan, Pare, \& Munhall, 2007). Intriguingly, deaf people also tend to look at the eyes of others communicating with sign language, although beginners also look at the mouth (Emmorey, Thompson, \& Colvin, 2009). The vast majority of these studies displayed the face of a single speaker performing a monologue, but we can extend the importance of gaze to the eyes to more realistic, three-party conversations.

In research involving interactive communication, Richardson, Dale, and Kirkham (2007) have documented the "gaze-coordination" in a conversation: conversants tend to look at the same thing at the same time. Other descriptions of the role of gaze in conversation suggest that it functions as a social signal for whose turn it is to talk next (Kendon, 1967). In our own analysis, we found that observers were quite likely to look at the person talking at any one moment (and most of the time this was at their eyes), but that gaze tended to predict the change from one speaker to the next. A similar finding was recently reported by von Hofsten, Uhlig, Adell, and Kochukhova (2009), who analysed the proportion of saccades that went from one speaker to the other within $2 \mathrm{~s}$ of the change in speaker. This study found that normally functioning children made these turn-tasking gaze shifts frequently, but that they were significantly less common in children with autistic spectrum disorder.

In our study, we used cross correlation to quantify the temporal lag between gaze and speech, and we found that the observing participants tended to look at targets around $150 \mathrm{~ms}$ before they spoke. Obviously our participants did not have the opportunity to actually converse with the targets, but it may be that the temporal pattern in gaze shifts reflects the general pattern of turn taking during a conversation. It is also interesting to consider the pattern of gaze preceding speech in the context of research into eye movements in speech perception. When participants listen to spoken sentences they recognize objects named in the sentence and move their eyes to these objects a few hundred milliseconds after the beginning of the word (Allopenna, Magnuson, \& Tanenhaus, 1998). In their study of gaze coordination, Richardson and Dale (2005) found that when viewing a picture of characters from a TV show, a listener's eye movements were best aligned with those of the speaker after a 2 s delay. In these studies, presumably, participants take time to process what they hear and plan appropriate eye movements. Why did we find that gaze preceded the speaker? One reason may be the dynamic and social nature of our targets. The targets were talking about abstract items, and we found a temporal synchrony with fixations to the speaker, rather than to any explicitly mentioned object. In fact, the pattern we observe is more 
similar to that found in studies of speech production, where people describing a scene tend to look at objects up to a second prior to naming them (Griffin \& Bock, 2000). Similarly, if the pragmatic or linguistic context of speech is predictive of what will be mentioned next, participants make anticipatory eye movements that precede the utterance (Altmann \& Kamide, 2009), something that also occurs in realistic interactions with a partner (Brown-Schmidt \& Tanenhaus, 2008). We suggest that the pattern we observed demonstrates the social context of the conversation to which our observers were attuned. That participants' gaze predicted who was going to speak next may indicate that the next speaker was being addressed or referred to by the current speaker (and so the observer may have been looking to observe their reaction), or that the context constrained who was going to speak next in other ways. This intriguing finding merits further study.

In conclusion, we have used a complex, realistic and social stimulus to explore the allocation of gaze in a group interaction. The people in this interaction, and in particular their eye regions, were potent targets for fixation. However, high-status individuals were looked at more often and for longer than low-status targets, which is consistent with a rapid perception of the social hierarchy in the scene and an evolutionarily determined bias toward attending to some people more than others. Gaze was also temporally yoked to the conversation between the people. These findings are among the first to demonstrate the influence of a realistic social context and the hierarchy that goes with it on the topdown allocation of eye gaze, and they provide a way forward for researchers investigating social attention.

\section{Acknowledgements}

We are grateful for the helpful suggestions of two anonymous reviewers, and for the generous support of: a Commonwealth Fellowship from the Government of Canada to TF; a Social Science and Humanities Research Council (SSHRC) of Canada award to JC (File \#s767-2009-2108); a SSHRC Standard Research Grant \#410-2009-2458 and a Michael Smith Foundation for Health Research Scholar Award [CI-SCH-01862(07-1)] to JT; and a Natural Sciences and Engineering Research Council operating grant to AK.

\section{References}

Abramovitch, R. (1976). The relation of attention and proximity to rank in preschool children. In M. R. A. Chance \& R. R. Larsen (Eds.), The social structure of attention (pp. 153-176). London: Wiley.

Adolphs, R., Gosselin, F., Buchanan, T. W., Tranel, D., Schyns, P., \& Damasio, A. R. (2005). A mechanism for impaired fear recognition after amygdala damage. Nature, 433(7021), 68-72.

Allopenna, P. D., Magnuson, J. S., \& Tanenhaus, M. K. (1998). Tracking the time course of spoken word recognition using eye movements: Evidence for continuous mapping models. Journal of Memory and Language, 38(4), 419-439.

Altmann, G. T. M., \& Kamide, Y. (2009). Discourse-mediation of the mapping between language and the visual world: Eye movements and mental representation. Cognition, 111(1), 55-71.

Anderson, C., John, O. P., Keltner, D., \& Kring, A. M. (2001). Who attains social status? Effects of personality and physical attractiveness in social groups. Journal of Personality and Social Psychology, 81, 116-132.

Ballard, D., \& Sprague, N. (2005). Modeling the brain's operating system Brain, Vision, and Artificial Intelligence. In Proceedings (Vol. 3704, pp. 347-366).
Baron-Cohen, S. (1995). Mindblindness: An essay on autism and theory of mind. Cambridge (MA): MIT Press.

Berger, J., Rosenholtz, S. J., \& Zelditch, M. (1980). Status organizing processes. Annual Review of Sociology, 6, 479-508.

Birmingham, E., \& Kingstone, A. (2009). Human social attention: A new look at past, present, and future investigations. The Year in Cognitive Neuroscience, Annals of the New York Academy of Sciences, 118-140.

Birmingham, E., Bischof, W. F., \& Kingstone, A. (2008). Gaze selection in complex social scenes. Visual Cognition, 16(2-3), 341-355.

Boehm, C. (1993). Egalitarian society and reverse dominance hierarchy. Current Anthropology, 34, 227-254.

Brown-Schmidt, S., \& Tanenhaus, M. K. (2008). Real-time investigation of referential domains in unscripted conversation: A targeted language game approach. Cognitive Science, 32(4), 643-684.

Buchan, J. N., Pare, M., \& Munhall, K. G. (2007). Spatial statistics of gaze fixations during dynamic face processing. Social Neuroscience, 2(1), $1-13$.

Chance, M. R. A. (1967). Attention structure as basis of primate rank orders. Man, 2(4), 503-518.

Cheng, J. T., Tracy, J. L., Henrich, J., Foulsham, T. \& Kingstone, A., in preparation. The emergence of dominance and prestige hierarchies in unacquainted social groups.

Chudek, M., Heller, S., Birch, S., \& Henrich, J., submitted for publication. Prestige-biased learning in children: Attention from others as a cue for cultural transmission.

Dadds, M. R., El Masry, Y., Wimalaweera, S., \& Guastella, A. J. (2008) Reduced eye gaze explains "Fear Blindness" in childhood psychopathic traits. Journal of the American Academy of Child and Adolescent Psychiatry, 47(4), 455-463.

Dalton, K. M., Nacewicz, B. M., Johnstone, T., Schaefer, H. S., Gernsbacher, M. A., Goldsmith, H. H., et al. (2005). Gaze fixation and the neural circuitry of face processing in autism. Nature Neuroscience, 8(4), $519-526$

Emery, N. J. (2000). The eyes have it: The neuroethology, function and evolution of social gaze. Neuroscience and Biobehavioral Reviews, 24(6), 581-604.

Emmorey, K., Thompson, R., \& Colvin, R. (2009). Eye gaze during comprehension of American sign language by native and beginning signers. Journal of Deaf Studies and Deaf Education, 14(2), 237-243.

Findlay, J. M., \& Gilchrist, I. D. (2003). Active vision the psychology of looking and seeing. Oxford: OUP.

Foulsham, T., \& Underwood, G. (2007). How does the purpose of inspection influence the potency of visual saliency in scene perception? Perception, 36, 1123-1138.

Foulsham, T., \& Underwood, G. (2008). What can saliency models predict about eye movements? Spatial and sequential aspects of fixations during encoding and recognition. Journal of Vision, 8(6), 1-17.

Friesen, C. K., \& Kingstone, A. (1998). The eyes have it! Reflexive orienting is triggered by nonpredictive gaze. Psychonomic Bulletin \& Review, 5(3), 490-495.

Ghazanfar, A. A., Nielsen, K., \& Logothetis, N. K. (2006). Eye movements of monkey observers viewing vocalizing conspecifics. Cognition, 101(3), 515-529.

Griffin, Z. M., \& Bock, K. (2000). What the eyes say about speaking. Psychological Science, 11(4), 274-279.

Guastella, A. J., Mitchell, P. B., \& Dadds, M. R. (2008). Oxytocin increases gaze to the eye region of human faces. Biological Psychiatry, 63(1), 3-5.

Hasson, U., Nir, Y., Levy, I., Fuhrmann, G., \& Malach, R. (2004). Intersubject synchronization of cortical activity during natural vision. Science, 303(5664), 1634-1640.

Hayhoe, M., \& Ballard, D. (2005). Eye movements in natural behavior. Trends in Cognitive Sciences, 9(4), 188-194.

Henderson, J. M. (2003). Human gaze control during real-world scene perception. Trends in Cognitive Sciences, 7(11), 498-504.

Henrich, J., \& Gil-White, F. (2001). The evolution of prestige: Freely conferred status as a mechanism for enhancing the benefits of cultural transmission. Evolution and Human Behavior, 22, 1-32.

Itti, L. (2005). Quantifying the contribution of low-level saliency to human eye movements in dynamic scenes. Visual Cognition, 12(6), 1093-1123.

Itti, L., \& Koch, C. (2000). A saliency-based search mechanism for overt and covert shifts of visual attention. Vision Research, 40(10-12), 1489-1506.

Kendon, A. (1967). Some functions of gaze-direction in social interaction. Acta Psychologica, 26(1), 22.

Kingstone, A. (2009). Taking a real look at social attention. Current Opinion in Neurobiology, 19(1), 52-56.

Klin, A., Jones, W., Schultz, R., Volkmar, F., \& Cohen, D. (2002). Visual fixation patterns during viewing of naturalistic social situations as 
predictors of social competence in individuals with autism. Archives of General Psychiatry, 59(9), 809-816.

Kobayashi, H., \& Koshima, S. (1997). Unique morphology of the human eye. Nature, 387, 767-768.

LaFreniere, P. J.. \& Charlesworth, W. R. (1983). Dominance, attention, and affiliation in a preschool group: A 9-month longitudinal study. Ethology and Sociobiology, 4, 55-67.

Land, M. F., \& Hayhoe, M. (2001). In what ways do eye movements contribute to everyday activities? Vision Research, 41, 3559-3566.

Li, N. P., Bailey, J. M., Kenrick, D. T., \& Linsenmeier, J. A. W. (2002). The necessities and luxuries of mate preferences: Testing the tradeoffs. Journal of Personality and Social Psychology, 82, 947-955.

Maner, J. K., DeWall, C. N., \& Gailliot, M. T. (2008). Selective attention to signs of success: Social dominance and early stage interpersonal perception. Personality and Social Psychology Bulletin, 34(4), 488-501.

Maner, J. K., Kenrick, D. T., Becker, D. V., Delton, A. W., Hofer, B., Wilbur, C. J., et al. (2003). Sexually selective cognition: Beauty captures the mind of the beholder. Journal of Personality and Social Psychology, 85(6), $1107-1120$.

McNelis, N. L., \& Boatright-Horowitz, S. L. (1998). Social monitoring in a primate group: The relationship between visual attention and hierarchical ranks. Animal Cognition, 1, 65-69.

Pylyshyn, Z. W., \& Storm, R. W. (1988). Tracking multiple independent targets: Evidence for a parallel tracking mechanism. Spatial Vision, 3, 179-197.

Raymond, J. E., Shapiro, K. L., \& Arnell, K. M. (1992). Temporary suppression of visual processing in an Rsvp task - An attentional blink. Journal of Experimental Psychology-Human Perception and Performance, 18(3), 849-860.

Richardson, D. C., \& Dale, R. (2005). Looking to understand: The coupling between speakers' and listeners' eye movements and its relationship to discourse comprehension. Cognitive Science, 29(6), 1045-1060.
Richardson, D. C., Dale, R., \& Kirkham, N. Z. (2007). The art of conversation is coordination - Common ground and the coupling of eye movements during dialogue. Psychological Science, 18(5), 407-413.

Riggio, R. E., Widamen, K. F., Tucker, J. S., \& Salinas, C. (1991). Beauty is more than skin deep: Components of attractiveness. Basic and Applied Social Psychology, 12, 423-439.

Rogers, R. D., \& Monsell, S. (1995). Costs of a predictable switch between simple cognitive tasks. Journal of Experimental Psychology-General, 124(2), 207-231.

Shepherd, S. V., Deaner, R. O., \& Platt, M. L. (2006). Social status gates social attention in monkeys. Current Biology, 16(4), R119-R120.

Shepherd, S. V., \& Platt, M. L. (2008). Spontaneous social orienting and gaze following in ringtailed lemurs (Lemur catta). Animal Cognition, 11(1), 13-20.

Tomasello, M., Carpenter, M., Call, J., Behne, T., \& Moll, H. (2005). Understanding and sharing intentions: The origins of cultural cognition. Behavioral and Brain Sciences, 28, 675-691.

Vatikiotis-Bateson, E., Eigsti, I. M., Yano, S., \& Munhall, K. G. (1998). Eye movement of perceivers during audiovisual speech perception. Perception \& Psychophysics, 60(6), 926-940.

Vaughn, B. E., \& Waters, E. (1981). Attention structure, sociometric status, and dominance: Interrelations, behavioral correlates, and relationships to social competence. Developmental Psychology, 17, 275-288.

von Hofsten, C., Uhlig, H., Adell, M., \& Kochukhova, O. (2009). How children with autism look at events. Research in Autism Spectrum Disorders, 3(2), 556-569.

Wilkowski, B. M., Robinson, M. D., \& Friesen, C. K. (2009). Gaze-triggered orienting as a tool of the belongingness self-regulation system. Psychological Science, 20, 495-501.

Yarbus, A. L. (1967). Eye movements and vision. New York: Plenum. 